\title{
Video Call Traffic Identification based on Bayesian Model
}

\author{
Ying Hou ${ }^{1, a}$, Hai Huang, Kai Wang, Yu-hang Zhu \\ ${ }^{1}$ PLA Information and Engineering University, ZhengZhou CHINA \\ andschhh@sina.com
}

Keywords: traffic identification, VolP, Bayesian, probability density

\begin{abstract}
This paper proposes Bayesian statistical method to identify the video traffic by the symmetrical features and coding statistical characteristics of video calls. According to the problem of high computational complexity of the non-parametric probability density estimate method in the condition of large samples, we propose grid probability density estimation method of gird division to reduce the computational complexity. We present identification results. The experimental results indicate that that this method can effectively detect video call traffic.
\end{abstract}

\section{Introduction}

With the development of IP voice technology and the increase of network bandwidth, VoIP services have developed rapidly. High profits and low costs of VoIP services making a large number of illegal VoIP operators flooded the telecommunications market, not only harm the economic interests of legitimate operators, but also break the competition pattern of the original telecom market. In order to effectively manage VoIP calls, the researchers conducted a lot of research on VoIP traffic identification, and have yielded some results. Currently, the user needs to further improve the communication experience and video calls become more and more popular. Therefore, it's important to identify Internet video call traffic for the effective management from the perspective of network management and traffic monitoring.

\section{Related Work}

Traffic identification is associating traffic flows with the applications — or application types that generated them. VoIP communication is multi- channel application. One VoIP call includes establishing, consulting and transferring of multiple session channels, for examples, control channel and media channel. The control channel is used for the establishment, release, media transmission parameter negotiation, media transmission process control of VoIP communication. And media channel is used for the exchange of audio or video data between the endpoints of VoIP communications. The VoIP traffic in this paper refers to the traffic of VoIP media channel including audio and video data.

Common methods used for VoIP traffic identification are traffic identification method based on the call protocol process analysis and identification method based on the statistical characteristics of the flow.

Traffic identification method based call protocol process includes the following process: first get the VoIP control channel flow. According to the call control protocol from the data analysis, extract the media session information of the call. And then identify the call media flow. The problem with this method is that can only recognize VoIP session using standard VoIP protocol such as SIP, H.323.It is out of action when the VoIP session using private protocols.

Traffic identification method based on the statistical characteristics of the flow judge with different traffic statistical characteristics manifested in different applications, not depended on the load content detection. Literature [1] identify VoIP traffic by detecting average packet interval and average packet size of a flow, according to the packet length characteristic and time characteristic of the VoIP audio code. Literature [2] extract the conversation mode of two-way flow and identify VoIP traffic by machine-learning approaches according to the normal interactive behavior of VoIP calls. 
Literature [3]-[5] research how to identify Skype traffic. However, these studies were only for audio calls, and not apply to video call traffic identification.

\section{Video Call Flow Characteristic}

Audio and video calls are real-time services. Throughout the session, both sides transfer audio and video data in the same encoding format. Therefore, from a statistical sense, the two flows of audio and video calls are symmetrical. The traffic of Web browsing, video-on-demand and FTP file transfer application presents a serious asymmetry because of the client - server model. The client sends a request to the server, and the server sends large amounts of data to the client. So traffic sent by the server is more than the client. In P2P service, the hosts upload when downloading. But in most of the P2P applications, in order to ensure the download rate, upload rate is limited, usually much smaller than the download rate. the P2P traffic shows asymmetric characteristics. Therefore, flow symmetry is the main features to different audio and video calls from other non-real-time interactive services.

For the real-time requirements of audio transmission is higher than video, audio coding schemes are usually use small packets and low packet interval and video coding schemes are usually use long packets. Such as G.711, the encoding rate is $64 \mathrm{kbps}$ and the payload length is 160 bytes. G.723, the encoding rates are $6.3 \mathrm{kbps}$ and $5.3 \mathrm{kbps}$. G.729, the encoding rate is $8 \mathrm{kbps}$, payload length is about 30 bytes. H.263, used in video encoding, the length of most of packet is from 700 to 1300 bytes. Therefore traffic can further distinguished into audio and video by the average packet interval and packet length.

To detect the video call traffic, characteristic parameters are defined as follows:

Define 1 FBW (Flow Band Width): Assume during a period of given session, the total bits of the session streaming is b, the session length is T, and then FBW of the session flow is denoted:

$$
F B W=\frac{T}{b}
$$

Define 2 DFBWR (Dual Flow Band the Width Ratio): Assume during a period of given session, the session bandwidth of the calling is fbw_caller and the session bandwidth of the called is fbw_callee. Then DFBWR of the session flow is denoted:

$$
D F B W R=\frac{\text { flow_caller }}{\text { flow_caller }+ \text { flow_callee }}
$$

Define 3 MPAI (Mean the Packet Arrival Interval): Assume during a period of given session, the total number of packets is $\mathrm{n}$ and the moment of the ith packet arriving is $t_{i}$. Then MPAI of the session flow is denoted:

$$
M P A I=\frac{\sum_{i=1}^{n}\left(t_{n+1}-t_{n}\right)}{n}
$$

Define 4 VPAI (Variance Packet Arrival the Interval): Assume during a period of given session, the total number of packets is $\mathrm{n}$ and the moment of the ith packet arriving is $t_{i}$. Then VPAI of the session flow is denoted:

$$
V P A I=\sum_{i=1}^{n}\left(t_{n+1}-t_{n}-M P A I\right)^{2}
$$

Define 5 MPL (Mean Packet Length): Assume during a period of given session, the total number of packets is $n$ and the ith packet length is $l_{i}$. Then MPL of the session flow is denoted:

$$
M P L=\frac{\sum_{i=1}^{n} l_{n}}{n}
$$

Among them, DFBWR describes the symmetry properties of the flow and VPAI represents the coding features of the flow. 
In order to verify the characteristics of video call traffic, we collected call traffic of web, ftp, P2P download, the SIP voice terminals and SIP video terminals and compared the distinction of the characteristic parameters in these traffic. The results are shown in Fig.1 and Fig.2.
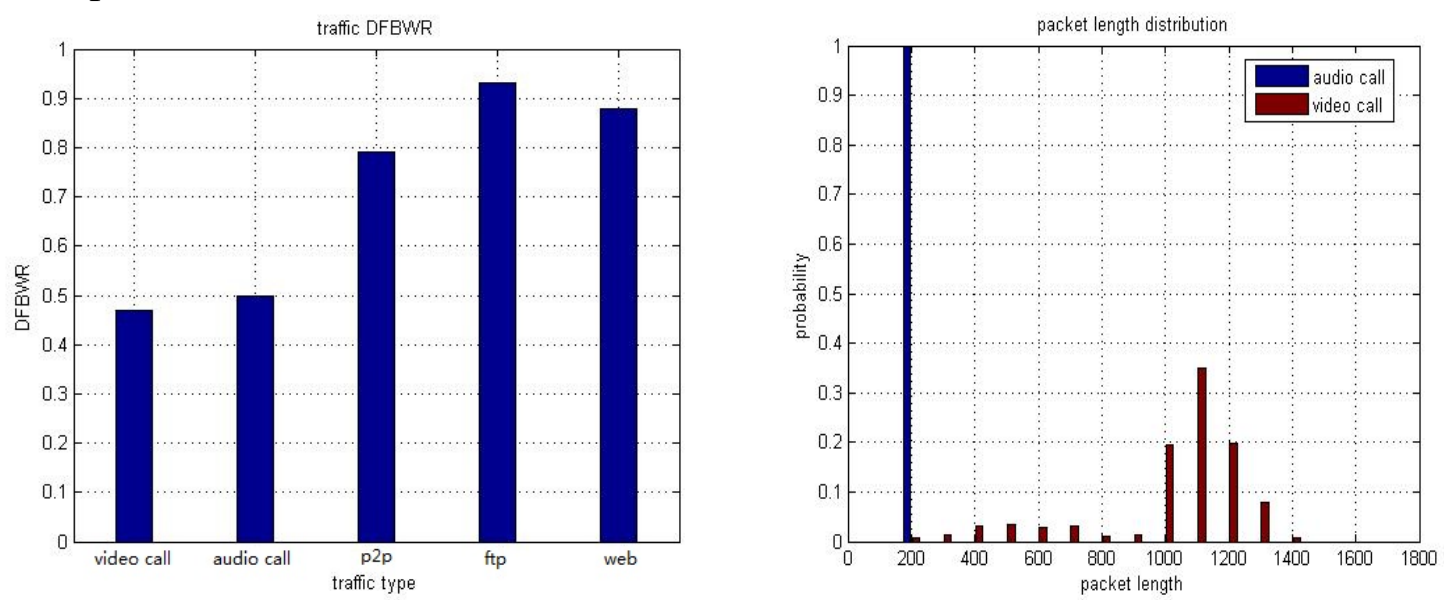

Fig. 1 DFBWR of some applications traffic

Fig. 2 audio and video call packet length distribution

\section{Bayesian Method}

In this paper, we use the above characteristic parameters derived to identify whether video traffic by Bayesian decision method.

Let $\left\{\omega_{1}, \omega_{2}\right\}$ denote VoIP and non-VoIP traffic, and $x=\{$ MPAI, VPAI, MPL, DFBWR $\}$ represent the normalization flow statistics characteristics vectors of the session flow. Let $p\left(x \mid \omega_{i}\right)$ denote the Class-conditional-probability density of $\mathrm{x}$ in the state of mode $\omega_{i}$. Let $P\left(\omega_{i}\right)$ denote the priori probability when the session flow mode is $\omega_{i}$. According to Bayesian formula, when a session flow statistics feature vector is $\mathrm{x}$, then posterior probability of the session in $\omega_{i}$ mode is given by:

$$
P\left(\omega_{j} \mid x\right)=\frac{p\left(x \mid \omega_{j}\right) P\left(\omega_{j}\right)}{\sum_{i=1}^{2} p\left(x \mid \omega_{i}\right) P\left(\omega_{i}\right)}
$$

$\lambda\left(\omega_{j} \mid \omega_{i}\right)$ denote the loss of a session flow mode $\omega_{i}$ misjudged as $\omega_{j}$. The risk judged as mode $\omega_{i}$, when the behavior characteristics vector is $\mathrm{x}$, can be expressed as:

$$
R\left(\omega_{i} \mid x\right)=\sum_{j=1}^{2} \lambda\left(\omega_{i} \mid \omega_{j}\right) P\left(\omega_{j} \mid x\right)
$$

The minimum risk is the last judgment.

According to the formula, we need to get the loss $\lambda\left(\omega_{j} \mid \omega_{i}\right)$, priori probability $P\left(\omega_{i}\right)$ and Class-conditional-probability density $p\left(x \mid \omega_{i}\right) . \lambda\left(\omega_{j} \mid \omega_{i}\right)$ is related to the actual classification problems and can be defined according to the actual needs in this paper. $P\left(\omega_{i}\right)$ can be estimated by statistics the session flow number during certain period of time. $p\left(x \mid \omega_{i}\right)$ can be obtained by probability density estimation method.

Define 4 Grid Collection: D dimensional space grid collection defined as $\Omega=\left\{G_{k}\right\}$, which:

$$
G_{k}=\left[\left(k-\frac{1}{2}\right) h,\left(k+\frac{1}{2}\right) h\right] \quad k \text { is integer, } h \text { is window width }
$$

According to the definition, the grid collection actually is the hypercube made up of every dimension in data space $\mathrm{R}$ divided by equal interval $\mathrm{h}$.

Define 5 Grid Probability Density: The probability density P of the grid $G_{k}$ is the probability density of the grid center point $\mathrm{x}$, and $P\left(G_{k}\right)=p(x), \quad x=k h$. 
Define 6 Grid Density Estimates: Define $f(x)$ as the probability density of the data points $x$ on a given space R. Define the function:

$$
f^{\prime}(x)=P\left(G_{x / h}\right)
$$

called grid density estimation of density function $f(x)$.

Obviously when $h$ is small enough, the probability density $f(x)$ of point $x$ infinitely close to the grid density estimation $f^{\prime}(x)$. According to the definition of the grid density estimates, its essence belongs to the kernel function estimation. the kernel function is defined as the following form:

$$
\psi(y)= \begin{cases}1, & \text { when } 0<y \leq 1 \\ 0, & \text { other }\end{cases}
$$

The grid probability density estimation converts the probability density of the data points to the simple calculation of grid probability density. And according to the definition of grid probability density, it can be obtained in advance based on data samples. And therefore for any one new sample, the calculation complexity of probability density estimation is $O(1)$. In addition, the grid probability density needs to save the number of samples in each grid, and need not to save the original data sample. That significantly saves the storage resources. The disadvantage is in order to ensure the accuracy of the estimation, the sample size required is relatively large.

\section{Identification Result}

We collected one day data for test from export link in the campus network, 522 calls using SIP video terminal in acquisition time. A part of data is used for training, another part set as the test data for verification. The evaluation of the algorithm consists of two: accuracy rate of NR and detection rate $\mathrm{PR}$.

The test data set for Bayesian model grid width from 0.1 to 0.3 , we get the test results of Bayesian classifier under different window width. As shown in the Fig. 3, when the grid width is equal to 0.22 , the integrated detection result is preferably. This is because in the case of a small sample too small width of the grid does not conducive to the estimation of the real probability density and too large width of the grid also easily lead to more estimation errors. Therefore, when the grid width is 0.22 , the algorithm will obtain the best detection results.

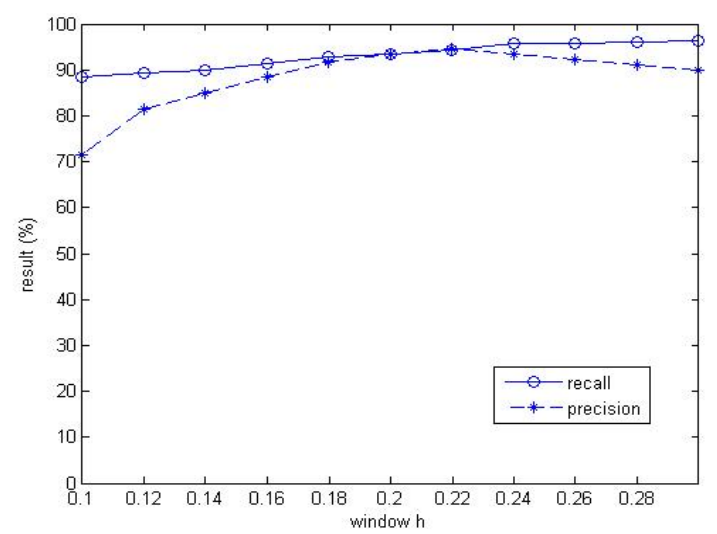

Fig.3 Identification accuracy of Bayesian method

\section{Summary}

This paper presents a video call traffic identification method based on Bayesian model. Using symmetrical features and coding statistical characteristics of real-time interactive traffic video calls, video call traffic is identified through the Bayesian statistical method. For computationally complexity of the non-parametric probability density estimate method of large sample, we proposed grid probability density estimation method to reduce the computational complexity. The test results show that this method can effectively detect video call traffic. 


\section{Acknowledgment}

Supported by National High Technology Research and Development Program of China(863 Program) (No. 2011AA010603 and No. 2011AA010605).

\section{References}

[1] Fauzia Idrees Uzma Aslam Khan. A Generic Technique for Voice over Internet Protocol (VoIP) Traffic Detection. IJCSNS International Journal of Computer Science and Network Security, VOL.8 No.2, February 2008,pp 52-59.

[2] Chen-Chi Wu, Kuan-Ta Chen, Yu-Chun Chang, Chin-Laung Lei. Detecting VoIP Traffic Based on Human Conversation Patterns. Principles, Systems and Applications of IP Telecommunications. Services and Security for Next Generation Networks Lecture Notes in Computer Science Volume 5310, 2008, pp 280-295.

[3] Sándor Molnár, Marcell Perényiet. On the identification and analysis of Skype traffic. International journal of communication systems. 2011; 24:94-117.

[4] Dongyan Zhang, Chao Zheng, Hongli Zhang, Hongliang Yu . Identification and Analysis of Skype Peer-to-Peer Traffic. Proc of 2010 Fifth International Conference on Internet and Web Applications and Services. Barcelona, 2010,pp 200-206.

[5] M. Perényi, A. Gefferth, T. D. Dang, and S. Molnár. Skype traffic identification. IEEE Globecom 2007, Washington, DC, USA, Nov.2007. 